
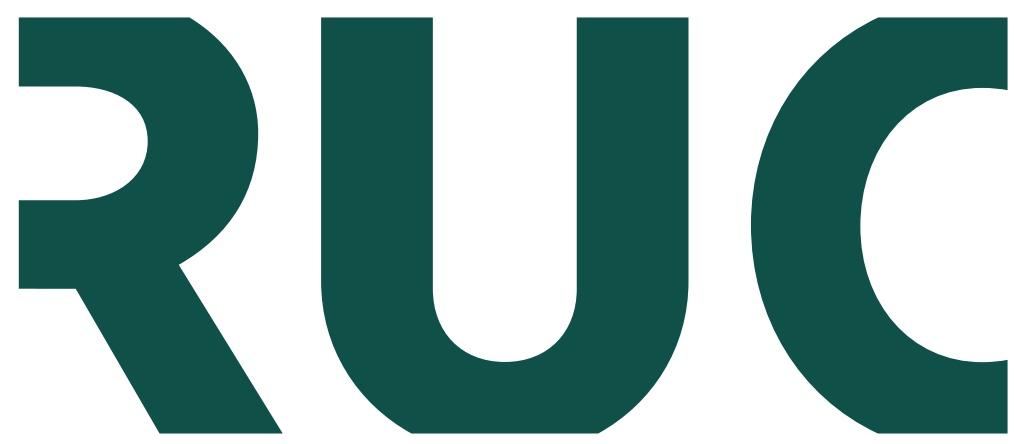

Roskilde University

\title{
Oscillatory shear and high-pressure dielectric study of 5-methyl-3-heptanol
}

Hecksher, Tina; Jakobsen, Bo; Dyre, J. C.; Gainaru, Catalin; Böhmer, Roland; Pawlus, S.; Paluch, M.; Figuli, R; Wilhelm, M

Published in:

Colloid and Polymer Science

DOI:

10.1007/s00396-014-3274-0

Publication date:

2014

Document Version

Peer reviewed version

Citation for published version (APA):

Hecksher, T., Jakobsen, B., Dyre, J. C., Gainaru, C., Böhmer, R., Pawlus, S., Paluch, M., Figuli, R., \& Wilhelm, M. (2014). Oscillatory shear and high-pressure dielectric study of 5-methyl-3-heptanol. Colloid and Polymer Science, 292(8), 1913-1921. https://doi.org/10.1007/s00396-014-3274-0

\section{General rights}

Copyright and moral rights for the publications made accessible in the public portal are retained by the authors and/or other copyright owners and it is a condition of accessing publications that users recognise and abide by the legal requirements associated with these rights.

- Users may download and print one copy of any publication from the public portal for the purpose of private study or research.

- You may not further distribute the material or use it for any profit-making activity or commercial gain.

- You may freely distribute the URL identifying the publication in the public portal.

Take down policy

If you believe that this document breaches copyright please contact rucforsk@kb.dk providing details, and we will remove access to the work immediately and investigate your claim. 


\title{
Oscillatory shear and high-pressure dielectric study of 5-methyl-3-heptanol
}

\author{
C. Gainaru • M. Wikarek • S. Pawlus • M. Paluch • \\ R. Figuli • M. Wilhelm • T. Hecksher • B. Jakobsen • \\ J. C. Dyre $\cdot$ R. Böhmer
}

Received: 31 March 2014 /Revised: 13 May 2014 / Accepted: 13 May 2014 / Published online: 15 June 2014

(C) Springer-Verlag Berlin Heidelberg 2014

\begin{abstract}
The monohydroxy alcohol 5-methyl-3-heptanol is studied using rheology at ambient pressure and using dielectric spectroscopy at elevated pressures up to $1.03 \mathrm{GPa}$. Both experimental techniques reveal that the relaxational behavior of this liquid is intermediate between those that show a large Debye process, such as 2-ethyl-1-hexanol, or a small Debyelike feature, such as 4-methyl-3-heptanol, with which comparisons are made. Various phenomenological approaches assigning a time scale for the rheological signature of supramolecular dynamics in monohydroxy alcohols are discussed.
\end{abstract}

Keywords Rheology · Dielectric spectroscopy $\cdot$ Hydrogen bonds $\cdot$ Monohydroxy alcohols

\section{Introduction}

As powerful experimental techniques, broad band dielectric and shear mechanical spectroscopies are well known to provide important insights into the dynamics of complex liquids

This is a special issue in honor of Prof. Dr. Friedrich Kremer on the occasion of his 65 th birthday.

C. Gainaru $(\bowtie) \cdot$ R. Böhmer

Fakultät Physik, Technische Universität Dortmund,

44221 Dortmund, Germany

e-mail: catalin.gainaru@uni-dortmund.de

M. Wikarek $\cdot$ S. Pawlus $\cdot$ M. Paluch

Institute of Physics, University of Silesia, ul. Uniwersytecka 4,

40-007 Katowice, Poland

R. Figuli $\cdot$ M. Wilhelm

Karlsruher Institut für Technologie, 76128 Karlsruhe, Germany

T. Hecksher • B. Jakobsen - J. C. Dyre

DNRF Centre "Glass and Time", IMFUFA, Department of Sciences,

Roskilde University, Postbox 260, DK-4000 Roskilde, Denmark
[1-3]. As interesting examples of such liquids, the Debye-like relaxation not only in monohydroxy alcohols [4] but also in other classes of supramolecular liquids [5-8] is currently receiving a great deal of attention. The insights obtained by studying these substances can also enhance the understanding of the properties of other (hydrogen-bonded) systems including water and bio-macromolecules. While the Debye-like relaxation was long believed to be experimentally accessible only via dielectric $[9,10]$ and related spectroscopies [11-13], recent investigations demonstrate that also nuclear magnetic resonance [14], oscillatory shear [15], quasi-elastic neutron scattering [16], and even structural studies [17] may be sensitive to this feature.

It is known since long that in these complex liquids, the strength of the Debye-like dielectric relaxation depends sensitively on the architecture of the constituting monohydroxy alcohol molecules, notably on the position of the hydroxyl group within them, with the isomeric heptanols and octanols being particularly thoroughly studied in this context [18-23]. Building upon seminal earlier work $[18,19]$, this sensitivity to the molecular structure was recently reemphasized in a highfield dielectric study of the series of $j$-methyl-3-heptanol $(j \mathrm{M} 3 \mathrm{H})$ liquids with $j=3,4,5$, and $6[24,25]$. In these liquids, only the position of the methyl group varies: it may sterically shield the $\mathrm{OH}$ group more-like in $3 \mathrm{M} 3 \mathrm{H}$ and $4 \mathrm{M} 3 \mathrm{H}$ for which a Debye-like dielectric feature is largely absent —or less-like in $6 \mathrm{M} 3 \mathrm{H}$. The latter substance displays an intense Debye peak, comparable to that of 2-ethyl-1-hexanol (2E1H), a monohydroxy alcohol that has recently attracted considerable interest [14, 15, 26-32].

It was suggested to classify these materials into group I or group II liquids depending on whether they possess a strong or a weak Debye-like process, respectively $[18,33]$. In other words, the question is whether the orientational correlation within the monohydroxy alcohol liquids as measured by the Kirkwood factor, $g_{\mathrm{K}}=1+z\langle\cos \theta\rangle$, is small or large [34]. Here, 
$\langle\cos \theta\rangle$ corresponds to the mean angle enclosed by dipole moment vectors averaged over a coordination shell containing $z$ molecules. Typically, one has $g_{\mathrm{K}}>1$ if mutually parallel dipolar alignment prevails, while $1>g_{\mathrm{K}}>0$ is often considered to arise from antiferroelectric or ring-like dipole correlations. Thus, $g_{\mathrm{K}}=1$ may either correspond to uncorrelated dipolar arrangements or occur if parallel and antiparallel relative orientations are suitable mixed.

In molecular terms, group I monohydroxy alcohols are thought to feature chain-like supramolecular association which, like in type A polymers [35-38], lead to sizeable (dielectric) normal modes, while for group II monohydroxy alcohols, ring-like multimeric structures are held responsible for a nearly complete cancelation of supramolecular contributions to the dielectric response.

The 5M3H liquid, which is in the focus of the present work, presents a particularly interesting intermediate case as is also evident from the dielectric loss spectra shown in Fig. 1. It is well documented, albeit not evident from Fig. 1, that the Kirkwood factor of $5 \mathrm{M} 3 \mathrm{H}$ is very small $\left(g_{\mathrm{K}} \approx 0.6\right)$ near ambient temperature [18] and that it reaches values close to $g_{\mathrm{K}} \approx 1.6$ upon approaching its glass transition temperature, $T_{\mathrm{g}}=162 \mathrm{~K}[24,25]$. Near $190 \mathrm{~K}$ and at ambient pressures, the Kirkwood factor $g_{\mathrm{K}}=1$ is crossed rather steeply. This was interpreted in terms of a conversion from ring-like to chainlike supramolecular structure upon cooling [18], but analogous effects can also be induced by application of large external electrical fields [24]. In the present article, we reexamine the $g_{\mathrm{K}}=1$ crossing of $5 \mathrm{M} 3 \mathrm{H}$ using high-pressure dielectric experiments, extending Johari and Dannhauser investigation [39] to higher pressures by a factor of more than 2 as well as to somewhat lower temperatures. These extensions are necessary to explore the intermediate character of $5 \mathrm{M} 3 \mathrm{H}$ more thoroughly.

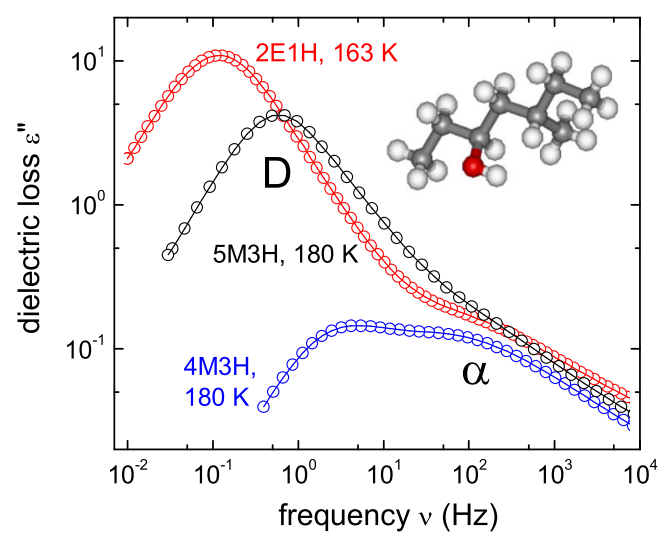

Fig. 1 Dielectric loss spectra of the octanols $4 \mathrm{M} 3 \mathrm{H}, 5 \mathrm{M} 3 \mathrm{H}$, and $2 \mathrm{E} 1 \mathrm{H}$. The temperatures are chosen such that the dielectric $\alpha$-relaxation frequency is close to $100 \mathrm{~Hz}$. The strengths of the dielectric Debye process $(D)$ of the various liquids differ significantly, but not their $\alpha$-relaxation intensity. A sketch of the $5 \mathrm{M} 3 \mathrm{H}$ molecule is shown as inset
Variously pronounced supramolecular features were recently reported from rheological measurements on different monohydroxy alcohols [15]: While for 2E1H a shear mechanical spectrum similar to that of covalently bonded oligomers (of, e.g., polystyrene $[40,41]$ ) was obtained, the supramolecular rheological signature of 4M3H was rather faint. Hence, it is most interesting to explore whether or not an intermediate mechanical behavior is found using the shear response of $5 \mathrm{M} 3 \mathrm{H}$.

\section{Experimental details}

$5 \mathrm{M} 3 \mathrm{H}$ (purity $\geq 99 \%$ ) was purchased from Sigma-Aldrich and used as received. High-pressure dielectric experiments up to $1.03 \mathrm{GPa}$ were carried out in the frequency range from $10^{-1}$ to $10^{6} \mathrm{~Hz}$ using the setup described previously [42]. Measurements of the complex shear mechanical modulus, $G^{*}(\nu)=G^{\prime}(\nu)+\mathrm{i} G^{\prime \prime}(\nu)$, were conducted using an ARES G2 rheometer from TA Instruments, covering a frequency range from $10^{-1}$ to $100 \mathrm{~Hz}$, and a piezoelectric shear modulus gauge technique, covering a frequency range from $10^{-3}$ to $10^{4} \mathrm{~Hz}$, also earlier described $[15,43]$.

\section{Rheological experiments: results and analyses}

In Fig. 2a, we present the in-phase or storage component of the mechanical shear modulus, $G^{\prime}(\nu)$, of $5 \mathrm{M} 3 \mathrm{H}$. One recognizes that as a function of temperature and frequency, the magnitude of $G^{\prime}(\nu)$ changes by several orders of magnitude. In particular, the slope of the curves varies, and near temperatures of $170 \mathrm{~K}, G^{\prime}(\nu)$ turns flat. At the lowest $T$, this indicates the high-frequency shear modulus, $G_{\infty}$, which was used to normalize the data in Fig. 2a, b. Assuming that $G_{\infty}$ and the shape of the overall shear mechanical spectrum is essentially independent of temperature in the $20 \mathrm{~K}$ interval covered by the present experiment, one can collapse all the data onto a master curve. The corresponding procedure is illustrated in Fig. $2 b$ where the curves from Fig. 2a were shifted horizontally along the frequency scale in order to achieve best overlap, well known as frequency temperature superposition (FTS). Before turning to a discussion of the shift factors, let us emphasize that Fig. $2 b$ demonstrates nicely that the obtained master curve for $5 \mathrm{M} 3 \mathrm{H}$ is indeed intermediate between those previously published for 4M3H (black open triangles) and for 2E1H (black open circles dashed line) [15]. This result shows that the intermediate behavior seen in dielectric spectroscopy, cf. Fig. 1, is also borne out rheologically, further vindicating the assertion [15] that the strength of the dielectric Debye process is reflected in the intensity of its normal-mode-like mechanical signature as well. It is worthwhile to emphasize that this observation does not only largely confirm the 


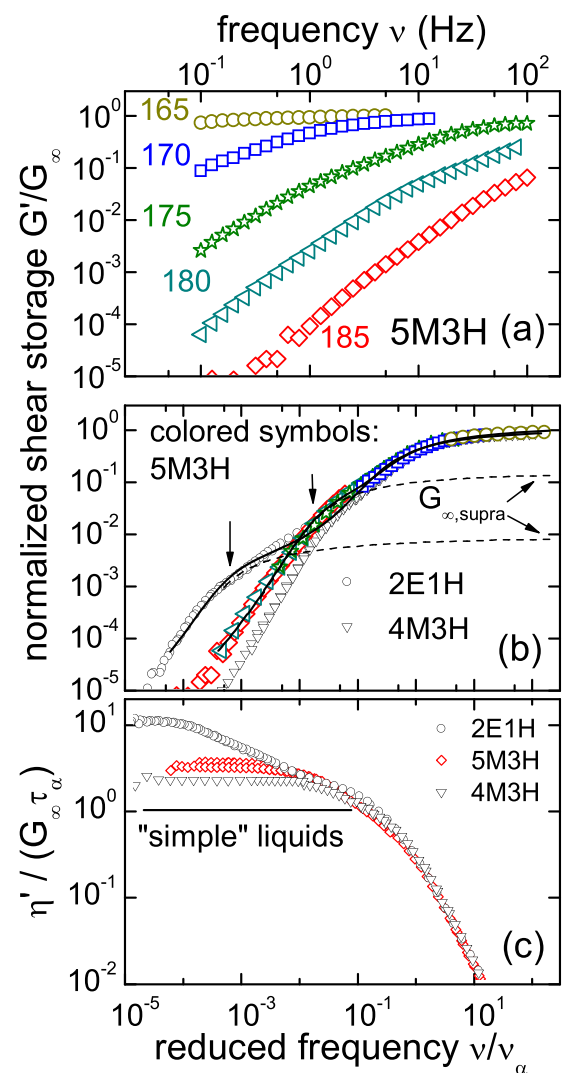

Fig. 2 a Normalized mechanical storage modulus, $G^{\prime}(\nu) / G_{\infty}$ of $5 \mathrm{M} 3 \mathrm{H}$. The numbers refer to temperatures in Kelvin. b Master curve obtained by shifting the data shown in frame (a) only along the frequency axis. The mechanical data of $4 \mathrm{M} 3 \mathrm{H}$ and $2 \mathrm{E} 1 \mathrm{H}$ are taken from ref. 15. The larger and smaller arrows indicate the estimated terminal relaxation modes for $2 \mathrm{E} 1 \mathrm{H}$ and $5 \mathrm{M} 3 \mathrm{H}$, respectively. The solid lines are interpolations of the $2 \mathrm{E} 1 \mathrm{H}$ and $5 \mathrm{M} 3 \mathrm{H}$ data using Eq. (1). The dashed lines highlight the spectral contribution of the supramolecular relaxation which for large $\nu$ saturates at $G_{\infty, \text { supra. }}$ c Dynamic shear viscosity $\eta^{\prime}=G^{\prime \prime} /(2 \pi \nu)$ normalized by the expectation from the Maxwell relation. One recognizes that also in this representation, $5 \mathrm{M} 3 \mathrm{H}$ displays an intermediate behavior. Furthermore, the results for the alcohols are compared to corresponding data (schematically represented as solid line) obtained for small-molecule glass formers devoid of a Debye process. For details and references to the original "simple" liquids data, see ref. 45-46

overlooked previous indications [44], but it supersedes previous beliefs that signatures of the Debye process are invisible to mechanical spectroscopy.

The shift factors obtained from the scaling procedure illustrated in Fig. $2 \mathrm{~b}$ can be exploited to evaluate the temperature dependence of the mechanical relaxation times. However, this holds only if frequency temperature superposition is applicable. A priori, it is not clear, however, whether this condition is fulfilled because we emphasized above that the supramolecular association in $5 \mathrm{M} 3 \mathrm{H}$ is subject to a relatively strongly temperature dependent ring-chain equilibrium. In Fig. 3, we collect the shift factors $\tau_{\text {rheo }}$ and compare them with the absolute time constants $\tau_{\text {PSG }}$ from measurements using the piezoelectric shear modulus gauge technique [43]. Excellent agreement of the time scales determined from both techniques is obtained. This demonstrates that the ring-chain equilibration does not affect the scaling procedure significantly. In hindsight, this can be rationalized by noting that the mechanical data were all recorded at temperatures much smaller than that corresponding to the $g_{\mathrm{K}}=1$ crossing at which the changes in the supramolecular structure are expected to be most pronounced.

In Fig. 2c, the shear data for the three monohydroxy alcohols, given in a viscosity representation, are compared with results for substances devoid of a (dielectric and/or mechanical) Debye process. These substances are here designated as "simple liquids," see ref.[45, 46] for details. For these liquids, the Maxwell relation connects the low-frequency viscosity, $\eta_{0}$, associated with the macroscopic flow with the relaxation time, $\tau_{\text {Maxwell, } 1}$, and the high-frequency shear modulus, $G_{\infty}$, according to $\eta_{0}=G_{\infty} \tau_{\text {Maxwell,1 } 1}$ [47]. Fig. 3 demonstrates that this relation, when applied to monohydroxy alcohols, provides time scales $\tau_{\text {Maxwell,1 }}$ that are slightly larger than $\tau_{\max }$ as obtained from the peak maximum in the shear loss, $G^{\prime \prime}(\nu)$. However, as is most obvious for the $2 \mathrm{E} 1 \mathrm{H}$ data in Fig. 2c, the main contribution to the static (or low shear rate) viscosity, $\eta(\nu \rightarrow 0)$, is provided by the supramolecular relaxation modes.

Thus, following considerations known from polymer science, one may estimate a mechanical relaxation time, $\tau_{\text {Maxwell,2}}$, if one replaces $G_{\infty}$ in the Maxwell relation by the much smaller shear modulus $G_{\infty, \text { supra }}$ associated with the supramolecular hydrogen-bonded aggregates, cf. Fig. $2 b$ $[48]^{1}$. $G_{\infty, \text { supra }}$ was determined by parameterizing the $G^{\prime}$ data using a sum of two Cole-Davidson functions $[49,50]$

$G^{\prime}(\nu)=\operatorname{Re}\left\{G_{\infty, \text { supra }}\left[1-\frac{1}{\left(1+2 \pi i \nu \tau_{\text {supra }}\right)^{\gamma_{\text {supra }}}}\right]+G_{\infty, \alpha}\left[1-\frac{1}{\left(1+2 \pi i \nu \tau_{\text {rheo }, \alpha}\right)^{\gamma_{\alpha}}}\right]\right\}$,

which describes the data quite well, see Fig. 2b. In Eq. (1), the two indices correspond to the supramolecular and the structural process, respectively, and the parameters $\gamma_{\text {supra }} \approx \gamma_{\alpha}=$ $0.25 \pm 0.05$ are measures for the width of the distribution of relaxation times underlying the different processes. The results for $\tau_{\text {Maxwell,1 }}=\eta_{0} / G_{\infty}$ and for $\tau_{\text {Maxwell,2 }}=\eta_{0} / G_{\infty, \text { supra }}$ are included in Fig. 3 and will be discussed further below.

\section{Dielectric high-pressure experiments: results and analyses}

In Fig. 4, we present dielectric loss spectra obtained at 265, 223 , and $203 \mathrm{~K}$ for various pressures ranging up to 1,031 MPa. One recognizes how, under isothermal conditions, the loss peaks shift to lower frequencies as the liquid is

\footnotetext{
${ }^{1}$ Here, we considered an additive approach $G_{\infty}=G_{\infty, \text { supra }}+G_{\infty, \alpha}$ similar to the one expressed by Eq. 8.8.6 in [48]
} 


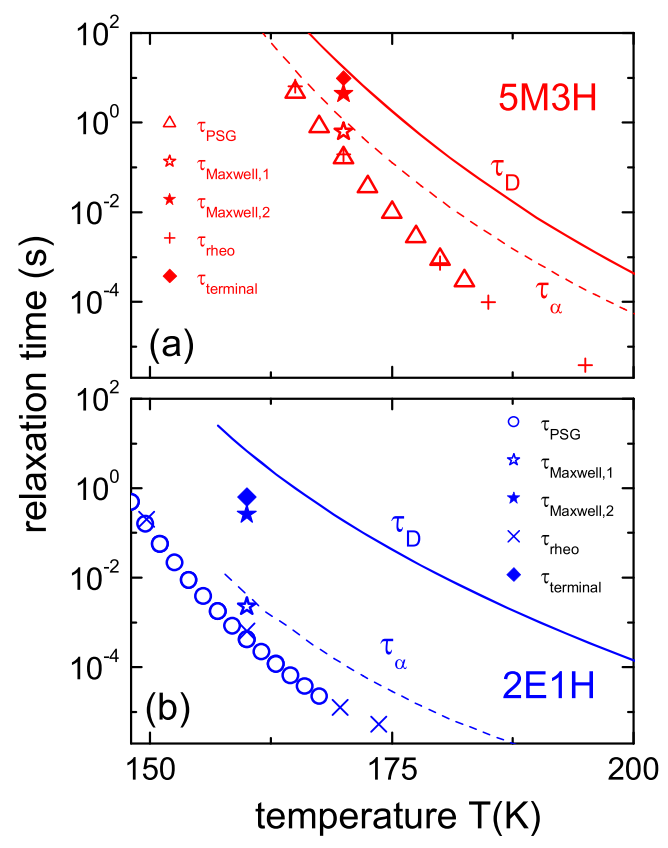

Fig. 3 Temperature-dependent shear relaxation times of a 5M3H (red triangles: PSG technique, current work; red crosses: rheometer, current work) and $\mathbf{b} 2 \mathrm{E} 1 \mathrm{H}$ (blue circles and blue crosses, taken from ref. 15). The diamonds reflect the time scales of the terminal relaxation modes indicated by the arrows in Fig. 2b. The stars are calculations based on the Maxwell relation: the open symbols refer to $\tau_{\text {Maxwell,1 }}=\eta_{0} / G_{\infty}$ and the filled symbols to $\tau_{\text {Maxwell,2 }}=\eta_{0} / G_{\infty, \text { supra }}$, respectively. The lines represent dielectric time constants for the $\alpha$ process (dashed lines) and for the Debye process (solid lines) taken from Refs. $25^{6}$

compressed. The data displayed in Fig. 4 (and another set obtained at $213 \mathrm{~K}$, not shown) as well as the spectra provided in Fig. 1 were fitted using a sum of a Debye (index D) and a Cole-Cole function (involving a shape parameter $\alpha)^{2}$ [51]

$\varepsilon *(\nu)=\varepsilon_{\infty}+\frac{\Delta \varepsilon_{\mathrm{D}}}{1+2 \pi i \nu \tau_{\mathrm{D}}}+\frac{\Delta \varepsilon_{\alpha}}{1+\left(2 \pi i \nu \tau_{\alpha}\right)^{\alpha}}$.

Here, $\tau_{\alpha}$ and $\tau_{\mathrm{D}}$ denote the relaxation times and $\Delta \varepsilon_{\alpha}$ and $\Delta \varepsilon_{\alpha, \mathrm{D}}$ the relaxation strengths of the dielectric $\alpha$ - and Debye processes, respectively, and $\varepsilon_{\infty}$ is the high-frequency contribution.

The relaxation strength and time scale parameters obtained from the fits to the 5M3H spectra are summarized in Fig. 5. In panels (a) and (b), we show the pressure dependence of $\tau_{\mathrm{D}}$ and $\tau_{\alpha}$, respectively. Where overlap exists, excellent agreement with data from Johari and Dannhauser is noted [39]. In Fig. 5, we reproduce the time scales which these authors obtained at 226.8, 239.6, 248.4, 256.6, and 267.4 K.

One recognizes that the slope, $\partial \log _{10} \tau / \partial p$, of the $\tau(p)$ curves decreases for increasing temperatures. In other

\footnotetext{
${ }^{2}$ Instead of a Cole-Cole, also the Cole-Davidson function and generalized approaches were tested. However, the Cole-Cole function gave consistently the most reliable results in particular also for the $265 \mathrm{~K}$ isotherm. The $\alpha$ was treated as a free parameter and was found typically found in the range from 0.4 to 0.8 .
}

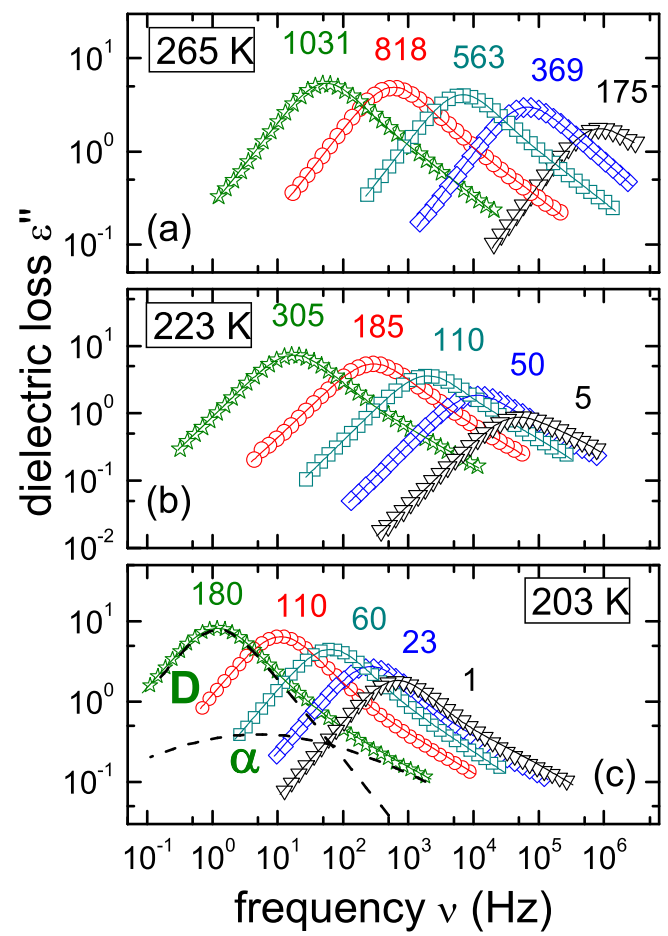

Fig. 4 Dielectric loss spectra of $5 \mathrm{M} 3 \mathrm{H}$ as a function of frequency measured isothermally for a $T=265 \mathrm{~K}, \mathbf{b} 223 \mathrm{~K}$, and $\mathbf{c} 203 \mathrm{~K}$ for several pressures (the numbers refer to pressures in megapascal). The lines are least-square fits using Eq. (2). For the dielectric loss measured at $203 \mathrm{~K}$ and $180 \mathrm{MPa}$, the individual contributions of Debye and $\alpha$-relaxation are highlighted by the dashed lines

words, with $R$ denoting the ideal gas constant, the activation volume [52]

$\Delta V_{\alpha, D}=\ln (10) R T\left(\frac{\partial \log _{10}\left(\tau_{\alpha, D} / \mathrm{s}\right)}{\partial p}\right)_{T}$

becomes smaller upon heating. Interestingly, for the $\alpha$ and the Debye process, the same activation volumes, $\Delta V=\Delta V_{\mathrm{D}} \approx \Delta V_{\alpha}$, are obtained within experimental error. This implies that $\tau_{\mathrm{D}} / \tau_{\alpha}$ should not depend on pressure. As a function of temperature, we find a linear decrease of $\Delta V$ from $65 \pm 8 \mathrm{~cm}^{3} / \mathrm{mol}$ (corresponding to $\sim 110 \AA^{3} /$ molecule) at $203 \mathrm{~K}$ to $25 \pm 5 \mathrm{~cm}^{3} / \mathrm{mol}$ (or $\sim 42 \AA^{3} /$ molecule) at $265 \mathrm{~K}$, similar to the values for $4 \mathrm{M} 3 \mathrm{H}$ [42]. For $5 \mathrm{M} 3 \mathrm{H}$, the pronounced temperature dependence of $\Delta V$ contrasts with the behavior of $2 \mathrm{E} 1 \mathrm{H}$. Here, from the data obtained in ref. 28 and reproduced in Fig. 5a, b, a temperatureindependent $\Delta V=22 \pm 3 \mathrm{~cm}^{3} / \mathrm{mol}$ is obtained in the 211 to $250 \mathrm{~K}$ range. For comparison, it is interesting to note that on the basis of a van der Waals radius of $3.33 \AA$ [53] for each of the isomeric octanol species, an equivalent sphere molecular volume of $\sim 95 \mathrm{~cm}^{3} / \mathrm{mol}\left(\sim 157.5 \AA^{3} /\right.$ molecule $)$ is theoretically estimated.

As summarized in Fig. 5c, different trends in the pressure dependence of the various alcohols are obtained for the strength ratio $\Delta \varepsilon_{D} / \Delta \varepsilon_{\alpha}$. While for $2 \mathrm{E} 1 \mathrm{H}$ [28] $\Delta \varepsilon_{D} / \Delta \varepsilon_{\alpha}$ decreases with 
increasing pressure and for 4M3H [42] this ratio increases with increasing pressure, for $5 \mathrm{M} 3 \mathrm{H}$, a relatively weak pressure dependence is found which in fact saturates beyond about $500 \mathrm{MPa}$.

This intermediate behavior of $5 \mathrm{M} 3 \mathrm{H}$ is not only borne out by $\Delta \varepsilon_{D} / \Delta \varepsilon_{\alpha}$, i.e., by a static quantity, but also by the time scale ratio $\tau_{D} / \tau_{\alpha}$. Under isothermal conditions, for $2 \mathrm{E} 1 \mathrm{H}$, this ratio increases upon compression; for $4 \mathrm{M} 3 \mathrm{H}$, it decreases; and for $5 \mathrm{M} 3 \mathrm{H}$, however, it is virtually pressure independent. These trends were already pointed out in ref. 42 taking data from ref. 39 into account. The present data extend the statement that the time scale ratio is constant $\left(\tau_{\mathrm{D}} / \tau_{\alpha}=8 \pm 3\right)$ up to pressures exceeding 1,000 MPa. More generally, these results confirm that increasing $\Delta \varepsilon_{D} / \Delta \varepsilon_{\alpha}$ ratios are typically associated with decreasing $\tau_{\mathrm{D}} / \tau_{\alpha}$ ratios. ${ }^{3}$

To express the changes of the mutual arrangement of the molecular dipoles, based on the dielectric constant $\varepsilon_{\mathrm{s}}$, it is useful to calculate the Kirkwood factor

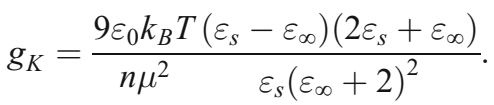

Here, $\mu=1.68 \mathrm{D}$ is the molecular dipole moment (assumed to be independent of pressure) and the number density, $n=\rho$ $N_{\mathrm{A}} / M$, is obtained from the pressure- and temperaturedependent density $\rho{ }^{4}$ Furthermore, $k_{\mathrm{B}}$ is Boltzmann's constant, $\varepsilon_{0}$ is the permittivity of free space, $M$ is the molar mass of $5 \mathrm{M} 3 \mathrm{H}$, and $N_{\mathrm{A}}$ denotes Avogradro's number. It is important to note that $\varepsilon_{\mathrm{s}}$ and $\varepsilon_{\infty}$ are the low- and high-frequency limits of the dielectric constants estimated from the fits to the overall $\varepsilon^{\prime}(v)$ spectra. ${ }^{5}$ The Kirkwood factors $g_{\mathrm{K}}$ thus calculated are presented in Fig. 6 and compared with results from previous dielectric studies of $5 \mathrm{M} 3 \mathrm{H}$ that focused on ambient [25] or high pressures [39].

A glance at Fig. 6 reveals that under high-pressure conditions, Kirkwood factors that agree excellently with previous determinations are found [39]. At the highest pressures and thus longest Debye relaxation times, $\tau_{\mathrm{D}}$, a trend toward saturation of $g_{\mathrm{K}}$ is obvious for the present data, in harmony with observation made in Fig. $5 \mathrm{c}$ for $\Delta \varepsilon_{D} / \Delta \varepsilon_{\alpha}$.

In previous investigations, the role of the $g_{\mathrm{K}}=1$ crossing was emphasized, as it indicates an equilibrium of molecular associates characterized by either $g_{\mathrm{K}}<1$ (ring-like structures) or $g_{\mathrm{K}}>1$ (chain-like structures) [18]. In isobaric studies, the temperature derivative $\left|d g_{\mathrm{K}} / d T\right|$ should thus be particularly large in order to change this ring-to-chain equilibrium sensitively by even small external perturbations; for $5 \mathrm{M} 3 \mathrm{H}$, it was found that $d g_{\mathrm{K}} / d T T_{g=1}$ is $-0.044 \times 10^{-3} \mathrm{~K}^{-1}$ at $T \approx 192 \mathrm{~K}$ [24]. In order to enable a direct comparison of the previous isobaric with the present

\footnotetext{
$\overline{3}$ Also, 2M3H conforms to this trend, see ref. 36.

${ }^{4}$ See Table V and Eq. 1 in ref. 20.

${ }^{5}$ In ref. 25, it was emphasized that particularly for $g_{\mathrm{K}}$ values smaller than about unity, their numerical values can sensitively depend on the choice of $\varepsilon_{\infty}$.
}

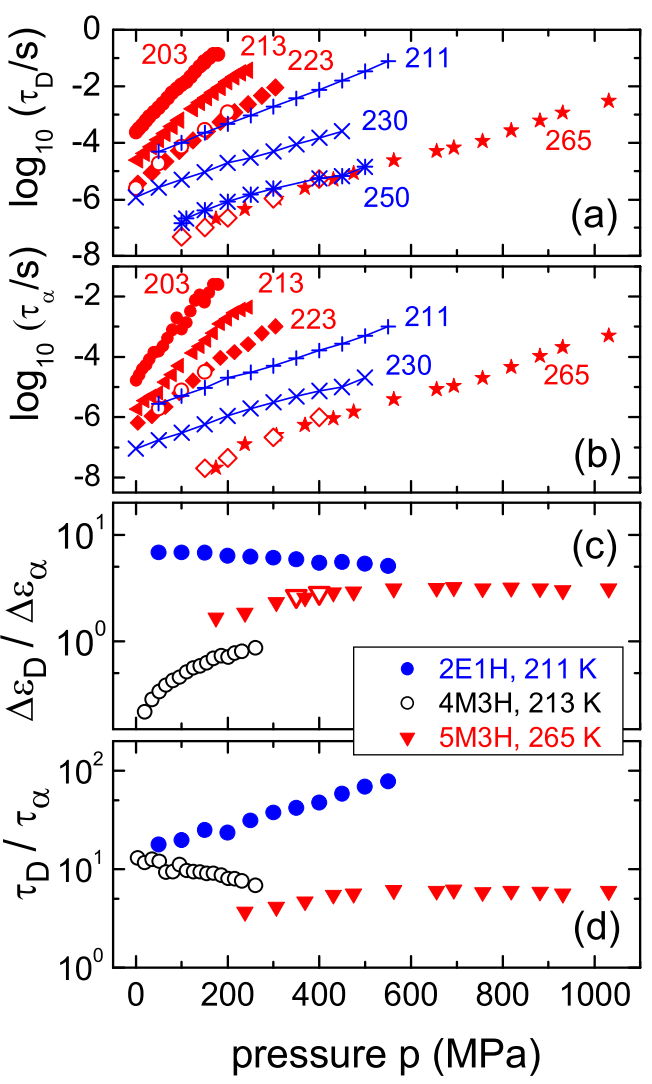

Fig. 5 a, $\mathbf{b}$ the pressure dependence of the relaxation times $\tau_{\mathrm{D}}$ and $\tau_{\alpha}$, respectively, obtained for $5 \mathrm{M} 3 \mathrm{H}$ from this work (filled symbols) and from Ref. 39 (circles for $T=226 \mathrm{~K}$ and open diamonds for $T=257 \mathrm{~K}$ ). Time scales for $2 \mathrm{E} 1 \mathrm{H}$ (blue lines and crosses, Ref. 28) are included for comparison. $\mathbf{c}, \mathbf{d}$ the pressure-dependent relaxation strength ratio, $\Delta \varepsilon_{D} /$ $\Delta \varepsilon_{\alpha}$, and time scale ratio, $\tau_{D} / \tau_{\alpha}$, respectively, are shown for $5 \mathrm{M} 3 \mathrm{H}$ from this work ( $265 \mathrm{~K}$, filled red triangles) and from Ref. 39 (266 K, open red triangles). The present data indicate that for $5 \mathrm{M} 3 \mathrm{H}, \Delta \varepsilon_{D} / \Delta \varepsilon_{\alpha}$ and $\tau_{D} / \tau_{\alpha}$ saturate at sufficiently high pressures. By contrast, the $\Delta \varepsilon_{D} / \Delta \varepsilon_{\alpha}$ ratios determined for 2E1H (blue dots, from Ref. 28) and 4M3H (black circles, from Ref. 42), both referring to lower temperatures, show decreasing and increasing ratios, respectively, as pressure is increased and opposite behavior is displayed by $\tau_{D} / \tau_{\alpha}$

high-pressure isothermal Kirkwood factors, like in a previous publication, see, e.g., Fig. 10 in ref. [23], we have chosen to represent $g_{\mathrm{K}}$ as a function of the Debye relaxation times $\tau_{\mathrm{D}}$.

\section{Discussion and concluding remarks}

Returning to the dielectric loss spectra shown in Fig. 1, it is remarkable that while the Debye (-like) process displays pronounced variations with molecular structure, the $\alpha$-relaxation strengths are largely independent of that structure when comparing them under iso- $\tau_{\alpha}$ conditions. The latter observation is easy to rationalize because any multimeric association does hardly affect the alcohol's intramolecular $\mathrm{C}-\mathrm{O}$ bond which essentially is responsible for those dipole moment contributions that are not directed along the contour of the supramolecular 


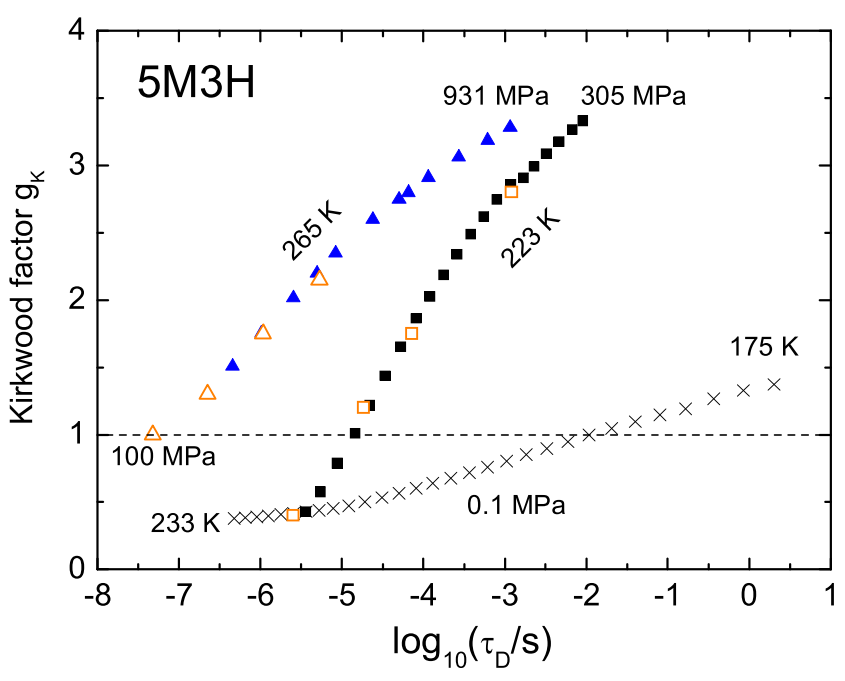

Fig. 6 Kirkwood factor $g_{\mathrm{K}}$ determined using Eq. (4) for 5M3H isothermally pressurized from this work (filled symbols) and from Ref. 39 (open symbols). Results obtained from temperature-dependent measurements at ambient pressure (crosses) are also included. These data are in good agreement with those from previous investigations [24]. The dashed line marks $g_{\mathrm{K}}=1$

structure $[14,54]$. Of course, if the electrical dipole moment is intentionally altered, e.g., by exploiting (in other materials) sulfur substitution along the alkyl chain of a monohydroxy alcohol, changes in the strength of the $\alpha$-relaxation are expected and were indeed observed experimentally [55].

The phenomenon that $g_{\mathrm{K}}=1$ is crossed while changing various external-state variables is not restricted to $5 \mathrm{M} 3 \mathrm{H}$. It is also shown by other neat alcohols such as, e.g., 2-methyl-2hexanol [23] and 2-butyl-1-octanol [17, 56]. A ring-to-chain equilibrium may also be expected to appear when mixing the ring-former $4 \mathrm{M} 3 \mathrm{H}$ with the chain-former $2 \mathrm{E} 1 \mathrm{H}$. Crossings of $g_{\mathrm{K}}=1$ with pronounced $d g_{\mathrm{K}} / d T$ slopes were indeed observed in an ambient-pressure study of mixtures of these two monohydroxy alcohols [32]. We wish to point out that whether $g_{\mathrm{K}}$ increases or decreases upon compression is not necessarily well defined for a given monohydroxy alcohol, but may depend on temperature as well. Here, 1-heptanol is an interesting example [22]: Below about $50{ }^{\circ} \mathrm{C}$, the total dielectric strength diminishes with increasing pressure, while above this temperature, it increases with increasing pressure.

Our current dielectric studies confirm that under isothermal conditions, the relation that an increasing $\Delta \varepsilon_{D} / \Delta \varepsilon_{\alpha}$ ratio is typically accompanied by a decreasing $\tau_{D} / \tau_{\alpha}$ ratio, see Fig. 5c, d. However, we have also observed that for $2 \mathrm{E} 1 \mathrm{H}$, with its large $\Delta \varepsilon_{D} / \Delta \varepsilon_{\alpha}$ and $\tau_{D} / \tau_{\alpha}$ ratios, a more pronounced mechanical low-frequency feature emerges.

Figure 3 compares the temperature-dependent structural relaxation times revealed by dielectric and rheological measurements for $2 \mathrm{E} 1 \mathrm{H}$ and $5 \mathrm{M} 3 \mathrm{H}$. Here, the open symbols represent the inverse frequency of the characteristic shear modulus loss peaks, and they appear smaller than the dielectric $\tau_{\alpha}$ (schematically plotted in Fig. 3 as dashed line), in accord with previous observations for monohydroxy alcohols [48] and other supercooled liquids [57].

Since the dielectric time constants for the Debye process $\tau_{D}$ are also available for $2 \mathrm{E} 1 \mathrm{H}$ and $5 \mathrm{M} 3 \mathrm{H}$ (see the solid lines in Fig. 3 representing data taken from refs. 25 and 48), it is worthwhile to compare them with various "characteristic" time scales associated with the low-frequency process that can be determined from the present shear relaxation experiments. On the one hand, most dielectric studies - including the present one-identify the Debye and the structural relaxation as distinct spectral processes [58], in accord with analyses of dielectric results on diluted monohydroxy alcohols, see, e.g., $[31,59,60]$. On the other hand, a standard procedure is yet to be established for parameterizing the spectral shape of the supramolecular rheological relaxation of monohydroxy alcohols, mainly due to the scarcity of experimental investigations accessing it.

The simplest approach may be to consider additivity of two rheological contributions, as expressed by Eq. (1). As demonstrated by Behrends and Kaatze, close to room temperature, the complete low-frequency flank of the ultrasonic absorption peak of 1-dodecanol can be well described by adding two monodispersive (Debye-like) processes [44]. However, when applied to the shear modulus response of deeply supercooled $2 \mathrm{E} 1 \mathrm{H}$, this simple procedure does not provide a satisfactory description (not shown). It is worth noting that the separation between the characteristic frequencies for the slow mode and the structural peak maximum is below one decade for 1decanol and other monohydroxy alcohols at room temperature at which the data in ref. 44 were taken, ${ }^{6}$ while for $2 \mathrm{E} 1 \mathrm{H}$ below $200 \mathrm{~K}$, this separation exceeds a factor of 1,000 , see Fig. $2 \mathrm{~b}$.

Many structural studies of monohydroxy alcohols indicate the formation of chain-like clusters. Therefore, one alternative is to adopt theoretical concepts applied to covalently bonded (polymeric) chains [15]. In this case, the time scales governing the supramolecular dynamics should be distributed. However, the viscosity or the characteristic time scale and amplitude of end-to-end dipole fluctuations are largely controlled by the terminal relaxation mode of the polymer, i.e., by a single time scale. Based upon this consideration, for $2 \mathrm{E} 1 \mathrm{H}$ and $5 \mathrm{M} 3 \mathrm{H}$, one may identify the terminal mode via the lowest frequency at which deviations from a simple power-law behavior occur in $G^{\prime}(\nu)$, see the arrows in Fig. 2b. The terminal time scales, $\tau_{\text {terminal }}$, thus estimated are plotted as full diamonds in Fig. 3. One observes that they are smaller, albeit not entirely different from $\tau_{D}$ as extracted on the basis of the dielectric spectra.

Finally, in Fig. 3, we also included the results obtained for $\tau_{\text {Maxwell,1 }}$ and $\tau_{\text {Maxwell,2 }}$ as open and filled stars, respectively. One recognizes that $\tau_{\text {Maxwell,2 }}$ is clearly larger than $\tau_{\text {Maxwell,1 }}$ (or $\tau_{\alpha}$ ), and it appears to be only slightly smaller than $\tau_{\text {terminal. }}$. All in all, the time scale of the terminal shear relaxation mode

\footnotetext{
${ }^{6}$ See Table 2 in ref. 44
} 
seems to provide the best agreement when compared with the time scale of the dielectric Debye process, in particular, when considering that often the shear dynamics is somewhat faster than the dielectric one [57].

To conclude, we have seen that in all respects examined in the current article, $5 \mathrm{M} 3 \mathrm{H}$ shows a behavior that is intermediate between that of monohydroxy alcohols with a strong Debye process (like $2 \mathrm{E} 1 \mathrm{H}$ ) or with a weak Debye-like feature (like 4M3H). This concerns the intensity of the viscoelastic normal mode, of the dielectric Debye process, as well as of the evolution of the dielectric relaxation strength and time scale ratio upon compression. For $5 \mathrm{M} 3 \mathrm{H}, \Delta \varepsilon_{D} / \Delta \varepsilon_{\alpha}$ and $\tau_{D} / \tau_{\alpha}$ are both relatively insensitive to high pressures when probed under isothermal conditions.

Acknowledgments Support of this project by the Deutsche Forschungsgemeinschaft under Grant No. BO1301/8-2 is gratefully acknowledged. S. P. and M. P. acknowledge the financial support of the project by the Polish National Science Centre on the basis of decision No. DEC-2012/05/B/ST4/00089. The centre for viscous liquid dynamics "Glass and Time" is sponsored by the Danish National Research Foundation's grant No. DNRF61.

\section{References}

1. McCrum NG, Read BE, Williams G (1967) Anelastic and dielectric effects in polymeric solids. Wiley, London

2. Kremer F, Schönhals A (eds) (2003) Broadband dielectric spectroscopy. Springer, Berlin

3. Ngai KL (2011) Relaxation and diffusion in complex systems. Springer, New York

4. Böhmer R, Gainaru C, Richert R (2014) Structure and dynamics of monohydroxy alcohols - milestones towards their microscopic understanding, 100 years after Debye. Phys Rep (submitted)

5. Lou N, Wang Y, Li X, Li H, Wang P, Wesdemiotis C, Sokolov AP, Xiong H (2013) Dielectric relaxation and rheological behavior of supramolecular polymeric liquid. Macromolecules 46:3160

6. Wang Y, Griffin PJ, Holt A, Fan F, Sokolov AP (2014) Observation of the slow, Debye-like relaxation in hydrogen-bonded liquids by dynamic light scattering. J Chem Phys 140:104510

7. Griffin PJ, Holt AP, Wang Y, Novikov VN, Sangoro JR, Kremer F, Sokolov AP (2014) Interplay between hydrophobic aggregation and charge transport in the ionic liquid methyltrioctylammonium bis(trifluoromethylsulfonyl)imide. J Phys Chem B 118:783

8. Wang L-M, Richert R (2005) Identification of dielectric and structural relaxations in glass-forming secondary amides. J Chem Phys 123:054516

9. Hansen C, Stickel F, Berger T, Richert R, Fischer EW (1997) Dynamics of glass-forming liquids. III. Comparing the dielectric $\alpha$ and $\beta$-relaxation of 1-propanol and o-terphenyl. J Chem Phys 107: 1086

10. Wang L-M, Richert R (2004) Dynamics of glass-forming liquids. IX. Structural versus dielectric relaxation in monohydroxy alcohols. J Chem Phys 124:11170

11. Wendt H, Richert R (1998) Purely mechanical solvation dynamics in supercooled liquids: the $\mathrm{S}_{0} \leftarrow \mathrm{T}_{1}(0-0)$ transition of naphthalene. $\mathrm{J}$ Phys Chem A 102:5775

12. Crossley J, Williams G. (1977) Relaxation in hydrogen-bonded liquids studied by dielectric and Kerr-effect techniques. J Chem Soc Faraday Trans 2, 73:1906
13. Coelho R, Khac Manh D (1967) Utilisation de la biréfringence électro-optique pour l'étude de la relaxation dipolaire dans les liquides polaires faiblement conducteurs. C R Acad Sci Paris Serie C 264:641

14. Gainaru C, Meier R, Schildmann S, Lederle C, Hiller W, Rössler EA, Böhmer R (2010) Nuclear magnetic resonance measurements reveal the origin of the Debye process in monohydroxy alcohols. Phys Rev Lett 105:258303

15. Gainaru C, Figuli R, Hecksher T, Jakobsen B, Dyre JC, Wilhelm M, Böhmer R (2014) Shear-modulus investigations of monohydroxy alcohols: evidence for a short-chain-polymer rheological response. Phys Rev Lett 112:098301

16. Sillrén P, Matic A, Karlsson M, Koza M, Maccarini M, Fouquet $P$, Götz M, Bauer T, Gulich R, Lunkenheimer P, Loidl A, Mattson J, Gainaru C, Vynokur E, Schildmann S, Bauer S, Böhmer R (2014) Liquid 1-propanol studied by neutron scattering, near-infrared, and dielectric spectroscopy. J Chem Phys 140:124501

17. Bierwirth SP, Büning T, Gainaru C, Sternemann C, Tolan M, Böhmer R (2014) Supramolecular X-ray signature of susceptibility amplification in hydrogen-bonded liquids. (preprint)

18. Dannhauser W (1968) Dielectric study of intermolecular association in isomeric octyl alcohols. J Chem Phys 48:1911

19. Dannhauser W (1968) Dielectric relaxation in isomeric octyl alcohols. J Chem Phys 48:1918

20. Johari GP, Dannhauser W (1968) Dielectric study of the pressure dependence of intermolecular association in isomeric octyl alcohols. J Chem Phys 48:5114

21. Dannhauser W, Flueckinger AF (1970) Liquid structure and dielectric relaxation of some isomeric methylheptanols. Phys Chem Liq 2:37

22. Vij JK, Scaife WG, Calderwood JH (1978) The pressure and temperature dependence of the static permittivity and density of heptanol isomers. J Phys D Appl Phys 11:545

23. Vij JK, Scaife WG, Calderwood JH (1981) The pressure and temperature dependence of the complex permittivity of heptanol isomers. J Phys D Appl Phys 14:733

24. Singh LP, Richert R (2012) Watching hydrogen bonded structures in an alcohol convert from rings to chains. Phys Rev Lett 109:167802

25. Singh LP, Alba-Simionesco C, Richert R (2013) Dynamics of glassforming liquids. XVII. Dielectric relaxation and intermolecular association in a series of isomeric octyl alcohols. J Chem Phys 139:144503

26. Huth H, Wang L-M, Schick C, Richert R (2007) Comparing calorimetric and dielectric polarization modes in viscous 2-ethyl-1hexanol. J Chem Phys 126:104503

27. Fragiadakis D, Roland CM, Casalini R (2010) Insights on the origin of the Debye process in monoalcohols from dielectric spectroscopy under extreme pressure conditions. J Chem Phys 132:144505

28. Reiser A, Kasper G, Gainaru C, Böhmer R (2010) High-pressure dielectric scaling study of a monohydroxy alcohol. J Chem Phys 132: 181101

29. Pawlus S, Paluch M, Dzida M (2010) Molecular dynamics changes induced by hydrostatic pressure in a supercooled primary alcohol. J Phys Chem Lett 1:3249

30. Schildmann S, Reiser A, Gainaru R, Gainaru C, Böhmer R (2011) Nuclear magnetic resonance and dielectric noise study of spectral densities and correlation functions in the glass forming monoalcohol 2-ethyl-1-hexanol. J Chem Phys 135:174511

31. Preuß M, Gainaru C, Hecksher T, Bauer S, Dyre JC, Richert R, Böhmer R (2012) Experimental studies of Debye-like process and structural relaxation in mixtures of 2-ethyl-1-hexanol and 2-ethyl-1hexyl bromide. J Chem Phys 137:144502

32. Bauer S, Wittkamp H, Schildmann S, Frey M, Hiller W, Hecksher T, Olsen NB, Gainaru C, Böhmer R (2013) Broadband dynamics in neat 4-methyl-3-heptanol and in mixtures with 2-ethyl-1-hexanol. J Chem Phys 139:134503

33. Johari GP (2013) Effects of electric field on the entropy, viscosity, relaxation time, and glass-formation. J Chem Phys 138:154503 
34. Kirkwood JG (1939) The dielectric polarization of polar liquids. J Chem Phys 7:911

35. Boese D, Kremer F (1990) Molecular dynamics in bulk cis-polyisoprene as studied by dielectric spectroscopy. Macromolecules 23:829

36. Baur ME, Stockmayer H (1965) Dielectric relaxation in liquid polypropylene oxides. J Chem Phys 43:4319

37. Adachi K, Kotaka T (1993) Dielectric normal-mode relaxation. Prog Polym Sci 18:585

38. Gainaru C, Hiller W, Böhmer R (2010) A dielectric study of oligoand poly(propylene glycol). Macromolecules 43:1907

39. Johari GP, Dannhauser W (1969) Effect of pressure on dielectric relaxation in isomeric octanols. J Chem Phys 50:1862

40. Gray RW, Harrison G, Lamb J (1977) Dynamic viscoelastic behaviour of low-molecular-mass polystyrene melts. Proc R Soc Lond A 356:77

41. Inoue T, Onogi T, Yao M-L, Osaki K (1999) Viscoelasticity of low molecular weight polystyrene. Separation of rubbery and glassy components. J Polym Sci Polym Phys 37:389

42. Pawlus S, Wikarek M, Gainaru C, Paluch M, Böhmer R (2013) How do high pressures change the Debye process of 4-methyl-3-heptanol? J Chem Phys 139:064501

43. Christensen T, Olsen NB (1995) A rheometer for the measurement of a high shear modulus covering more than seven decades of frequency below $50 \mathrm{kHz}$. Rev Sci Instrum 66:5019

44. Behrends R, Kaatze U (2001) Hydrogen bonding and chain conformational isomerization of alcohols probed by ultrasonic absorption and shear impedance spectrometry. J Phys Chem A 105:5829

45. Maggi C, Jakobsen B, Christensen T, Olsen NB, Dyre JC (2008) Supercooled liquid dynamics studied via shear-mechanical spectroscopy. J Phys Chem B 112:16320

46. Gainaru C, Hecksher T, Olsen NB, Böhmer R, Dyre JC (2012) Shear and dielectric responses of propylene carbonate, tripropylene glycol, and a mixture of two secondary amides. J Chem Phys 137:064508

47. Jakobsen B, Hecksher T, Christensen T, Olsen NB, Dyre JC, Niss K (2012) Communication: identical temperature dependence of the time scales of several linear-response functions of two glassforming liquids. J Chem Phys 136:081102

48. Strobl G (1997) The Physics of Polymers: Concepts for Understanding their Structures and Behaviour. Springer, Berlin

49. Davidson DW, Cole RH (1950) Dielectric relaxation in glycerine. J Chem Phys 18:1417

50. Deegan RD, Leheny RL, Menon N, Nagel SR, Venerus DC (1999) Dynamic shear modulus of tricresyl phosphate and squalane. J Phys Chem B 103:4066

51. Cole KS, Cole RH (1941) Dispersion and absorption in dielectrics-I alternating current characteristics. J Chem Phys 9:341

52. Roland CM, Hensel-Bielowka S, Paluch M, Casalini R (2005) Supercooled dynamics of glass-forming liquids and polymers under hydrostatic pressure. Rep Prog Phys 68:1405

53. Edward JT (1970) Molecular volumes and the Stokes-Einstein equation. J Chem Educ 47:261

54. Hassion FX, Cole RH (1953) Dielectric relaxation processes in ethanol. Nature 172:212

55. Gao Y, Bi D, Li X, Liu R, Tian Y, Wang L-M (2013) Debye-type dielectric relaxation in glass-forming 3-methylthio-1-hexanol. J Chem Phys 139:024503

56. Gao Y, Tu W, Chen Z, Tian Y, Liu R, Wang L-M (2013) Dielectric relaxation of long-chain glass-forming monohydroxy alcohols. J Chem Phys 139:164504

57. Jakobsen B, Niss K, Olsen NB (2005) Dielectric and shear mechanical alpha and beta relaxations in seven glass-forming liquids. J Chem Phys 123:234511

58. Davidson DW, Cole RH (1951) Dielectric relaxation in glycerol, propylene glycol, and n-propanol. J Chem Phys 19:1484
59. Power G, Vij JK, Johari GP (2007) Relaxations and nano-phaseseparation in ultraviscous heptanol-alkyl halide mixture. J Chem Phys 126:034512

60. Pawlus S, Paluch M, Dzida M (2011) Molecular dynamics changes induced by solvent in 2-ethyl-1-hexanol. Phys Rev E 84:031503

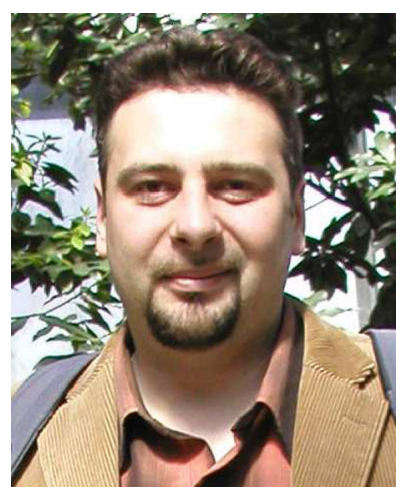

Catalin Gainaru studied medical physics at the Iasi University in Romania. His $\mathrm{PhD}$ thesis $(2008$, Prof. E. Rössler, University of Bayreuth) focused on the dielectric properties of low molecular weight liquids and glasses. $\mathrm{He}$ holds a postdoctoral position in the group of Prof. R. Böhmer at the Technical University of Dortmund, where he conducts dielectric, infrared, and rheological investigations. His research mainly concerns the dynamics in amorphous systems including suprastructural relaxation of polymers, a subject area which largely benefited from the expertise of Prof. F. Kremer.

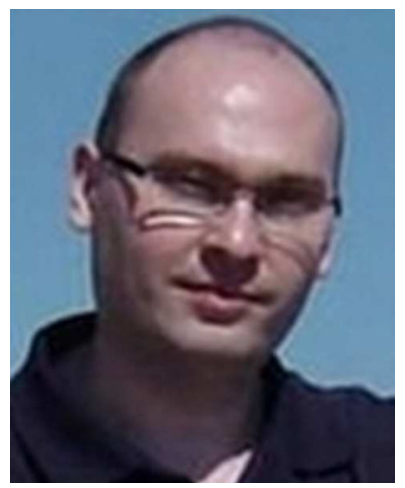

Sebastian Pawlus is assistant professor of physics at the University of Silesia. His major research interest are high pressure studies of the molecular dynamics of supramolecular structures in different materials e.g. monoand polyalcohols.

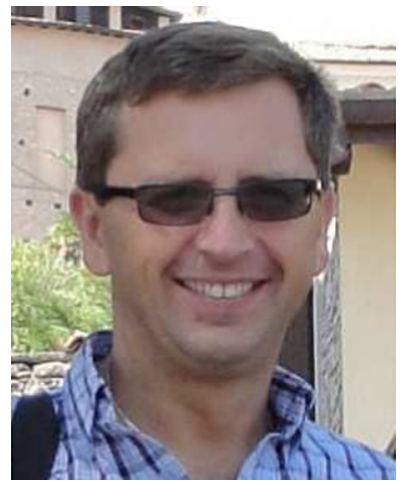

Marian Paluch is a professor of physics at the University of Silesia. His major research interest are high pressure studies on the molecular dynamics of complex systems. Coauthor of book: G. Floudas, M. Paluch, A. Grzybowski and K. L. Ngai; "Molecular Dynamics of GlassForming Systems - Effect of Pressure", Springer-Verlag 2010. Currently, he is collaborating with Prof. F. Kremer in the field of ionic liquids. 


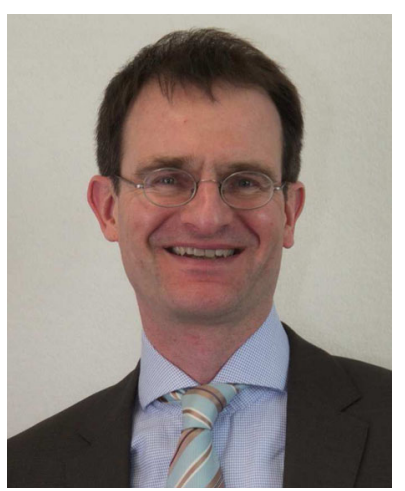

Manfred Wilhelm studied chemistry at the University of Mainz and Toronto. The PhD thesis (1995, H.W. Spiess) was on multidimensional Solid-State-NMR at the MPIPolymer in Mainz, where he got to know F. Kremer. 1995-1997 he was a postdoc at the Weizmann Institute in Israel. 1997 he returned to the MPI-P and finished habilitation about rheology in 2001. In 2004 he moved to the Technical University of Darmstadt as professor for polymer mechanics and continued 2006 at the KIT, Karlsruhe. One focus is the development of combined methods, like Rheo-Dielectrics, cooperating again with F. Kremer.

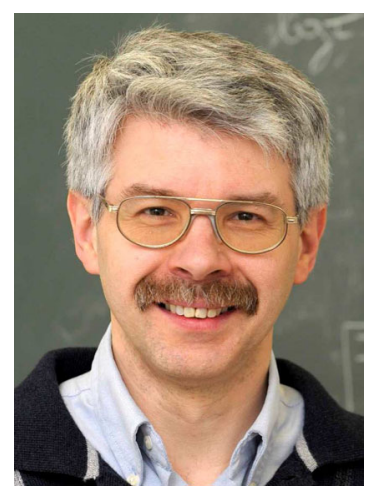

Roland Böhmer a professor at the TU Dortmund, studied Physics in Heidelberg and Mainz. During his diploma thesis in Mainz, in the group of Alois Loidl, he got to know and interacted with Frieder Kremer who worked two floors above in a different institute, but in the field of dielectric spectroscopy as well. Later, during postdoctoral positions he held in Tempe/Arizona, Darmstadt, and Mainz he turned also to other techniques like multidimensional NMR and recently rheology. His current research interests are in the fields of hydrogen-bonded liquids, the physics of amorphous and crystalline ices, as well as solid-state electrolytes. 